\title{
Prognostic significance of urine neutrophil gelatinase-associated lipocalin in patients with septic acute kidney injury
}

\author{
YONG GUO $^{1}$ and KUI-PO YAN ${ }^{2,3,4}$ \\ ${ }^{1}$ Neurosurgical Intensive Care Unit; ${ }^{2}$ Institute of Integrated Traditional Medicine and Western Medicine; \\ ${ }^{3}$ Key Unit of Traditional Chinese Medicine Gan of SATCM; ${ }^{4}$ Key Unit of the 11th Five-year Plan of SATCM \\ in Cerebrosis, Xiangya Hospital, Central South University, Changsha 410008, P.R. China
}

Received July 7, 2011; Accepted July 25, 2011

DOI: $10.3892 /$ etm.2011.339

\begin{abstract}
Acute kidney injury (AKI) is a common clinical problem which occurs in critically ill patients. Sepsis is now recognized as the most important contributing factor to AKI in this population. In clinical practice, certain studies have explored the urine neutrophil gelatinase-associated lipocalin (uNGAL) and the urine kidney injury molecule-1 (uKIM-1) as diagnostic and prognostic indices of AKI. Yet, it remains unclear whether UNGAL and UKIM-1 are associated with measures of disease severity and with adverse clinical outcomes in patients with established septic AKI of mixed cause and severity. Ninety-two septic patients with AKI were enrolled in a pilot study to test whether uKIM and UNGAL levels predict 180-day mortality. We initially performed univariate Cox proportional hazards analyses incorporating multiple demographic, clinical and laboratory variables. As a result, the APACHE II score $(\mathrm{p}=0.014)$ and $\mathrm{uNGAL}$ $(\mathrm{p}=0.015)$ were identified as independent predictors of 180-day mortality. On the other hand, there was no statistical difference in event-free survival between patients with and without higher serum creatinine, creatinine clearance and uKIM-1 (data not shown). In conclusion, uNGAL may be a promising predictor for septic patients with AKI, resulting in a clear increase in 180-day mortality. Further clinical evaluation of uNGAL is underway.
\end{abstract}

\section{Introduction}

Acute kidney injury (AKI), formerly known as acute renal failure, is a common clinical problem which occurs in critically ill patients (1-3). Sepsis is now recognized as the most important contributing factor to AKI in this population (3).

Correspondence to: Dr Yong Guo, Neurosurgical Intensive Care Unit, Xiangya Hospital, Central South University, Changsha 410008, P.R. China

E-mail: 568701692@qq.com

Key words: acute kidney injury, prognostic significance, urine neutrophil gelatinase-associated lipocalin, urine kidney injury molecule-1, APACHE II
For example, acute renal failure occurs in approximately $19 \%$ of patients with moderate sepsis, in $23 \%$ with severe sepsis and in $51 \%$ with septic shock when blood cultures are positive $(1,2)$. In the US, an estimated 700,000 cases of sepsis occur each year, resulting in more than 210,000 deaths (4). The combination of acute renal failure and sepsis is associated with a $70 \%$ mortality, as compared to a $45 \%$ mortality among patients with acute renal failure alone. Thus, the combination of sepsis and acute renal failure constitutes a particularly serious medical problem in the US (5).

In clinical practice, a reliable marker for the extent of AKI would be a valuable asset for the management of patients with septic AKI in the critical care setting. In adults, research has focused on the use of biochemical markers to detect and define the extent of AKI and to predict adverse outcome after AKI. These research findings have improved our understanding of the values of urinary markers for the early detection of AKI before there is a noticeable increase in serum creatinine. Regrettably, only a few clinical studies have focused on the prognostic utility of kidney injury markers in patients with established AKI.

Among the potential biochemical markers, certain studies have explored urine neutrophil gelatinase-associated lipocalin (uNGAL) and the urine kidney injury molecule-1 (uKIM-1) as diagnostic and prognostic indices of AKI. Human NGAL was originally identified as a $25-\mathrm{kDa}$ protein covalently bound to gelatinase from neutrophils (5-7). Although NGAL is expressed only at very low levels in several human tissues, it is markedly induced in injured epithelial cells, including the kidney (8). uKIM-1 is a more recently described type 1 cell membrane glycoprotein that is expressed in humans and rodents when the injured renal proximal tubule assumes a dedifferentiated phenotype (9). NGAL and KIM-1 are increased in urine very early $(2 \mathrm{~h})$ after injury, followed by IL-18 at $12 \mathrm{~h}$, and hence may serve as early detection biomarkers, at least in well-defined clinical settings. Both NGAL and KIM-1 performed well in predicting delayed graft function after renal transplantation; a study of both together may show significant synergy.

The aim of this study was to test the hypothesis that uNGAL and UKIM-1 are associated with measures of disease severity and with adverse clinical outcomes in patients with established septic AKI of mixed cause and severity. 


\section{Patients and methods}

Patient recruitment. Over the period from March 2008 to January 2009, we conducted a prospective observational study in intensive care units in Changsha, China. The study was approved by the institutional ethics committee. Informed consent was obtained from all patients or from their guardians prior to inclusion. We enrolled 92 septic patients with AKI and an expected stay in intensive care units (ICUs) of $>24 \mathrm{~h}$. Inclusion criteria were age $\geq 18$ years, AKI and sepsis. Exclusion criteria were prior kidney transplant, end-stage kidney disease (K/DOQI stages), renal replacement therapy (RRT) prior to ICU admission, or confirmed and/or suspected acute glomerulonephritis, interstitial nephritis, renal vasculitis or obstructive etiology for AKI. AKI was defined according to the risk-injury-failure-loss-ESRD (RIFLE) criteria (10). Worsening and/or progressive AKI was defined as transition from RIFLE class-risk to class-injury, failure or RRT initiation during the 5 days following enrollment. Sepsis was defined according to consensus guidelines (11). Shock was defined as a mean arterial pressure (MAP) $<60 \mathrm{mmHg}$ and/or need for vasoactive support. Preexisting co-morbid illnesses were defined by the Charlson index (12).

Clinical assessment and follow-up. Patients were identified by daily surveillance of the participating ICUs. Eligible patients underwent a medical record review. Data were extracted on standardized data forms. Clinical data included demographics, co-morbidities, mechanical ventilation, RRT details and clinical outcomes. Physiologic and laboratory data included weight, hemodynamics, vasoactive drugs, illness severity scores and details of pre-morbid and enrollment kidney function. With this information, the Acute Physiology and Chronic Health Evaluation II (APACHE II) score was calculated for all patients. All had indwelling urinary catheters. Patients had urine and blood drawn at enrollment. At the end of the hospital stay, the length of hospital stay and vital status were recorded. Finally, 180 days after AKI we contacted each patient's general practitioner to obtain information on vital status and hospital admissions. Our primary endpoint was 6-month mortality.

Laboratory methods. Urine samples for uNGAL testing were centrifuged at $1,500 \mathrm{rpm} \times 10 \mathrm{~min}$, and the supernatant was stored at $-70^{\circ} \mathrm{C}$ for batched analysis. uNGAL was measured by a chemiluminescent microparticle assay using the ARCHITECT platform (Abbott Diagnostics Inc., Abbott Park, IL, USA) (13). uKIM-1 measurements were performed using microspherebased Luminex xMAP technology with polyclonal antibodies raised against the human KIM-1 ectodomain. This technique is an adaptation of the sandwich ELISA assay. For measurements, 30- $\mu 1$ urine samples were analyzed in duplicate. Urinary creatinine levels were determined by a colorimetric method based on the Jaffe reaction (14). The urinary NGAL and KIM-1 levels were normalized to the urinary creatinine concentration and expressed as $\mathrm{ng} / \mathrm{mg}$ creatinine, to standardize and correct for changes in urine concentration. Four investigators blinded to the patients' clinical characteristics performed all of the urinary biomarker measures.

Statistical analysis. Analysis was performed using SPSS version 13.0 (SPSS Inc., Chicago, IL, USA). Continuous vari-
Table I. Main source of sepsis in patients with septic AKI.

\begin{tabular}{lr}
\hline Source of sepsis & No. $(\%)$ \\
\hline Intra-abdominal & $42(45.7)$ \\
Pulmonary thoracic & $29(31.5)$ \\
Skin/sofe tissue/bone & $13(14.1)$ \\
Urogenital & $8 \quad(8.7)$ \\
\hline
\end{tabular}

ables are expressed as the means \pm SD and categorical variables as frequency (\%). Comparision of parameters of the two groups (survivors and non-survivors) was carried out using the Student's t-test and the Fisher's exact test. uKIM-1 and UNGAL levels were adjusted for the APACHE II score since the APACHE II score represents a composite illness-severity score that takes into consideration several demographic, physiologic and laboratory variables, and the presence of sepsis. The performance characteristics of UNGAL levels and KIM-1 activity in predicting the composite outcome were described using the area under a receiver operator characteristic (ROC) curve and compared to the performance of more traditional clinical severity indices of kidney injury, including serum creatinine, urinary output and APACHE II score. Survival probabilities were calculated by the Kaplan-Meier method and comparisons were made using the log-rank test to identify potential prognostic factor. Variables found to be statistically significant at the $\mathrm{P}<0.05$ level were entered into a multivariate model using Cox regression analysis to identify the most statistically significant model. All $\mathrm{P}$ values are two sided, the level of significance is $\mathrm{P}<0.05$ and confidence intervals (CI) refer to $95 \%$ boundaries.

\section{Results}

The demographic and clinical characteristics of the 92 patients on inclusion are shown in Tables I and II. The cases of septic AKI were classified into two groups (survivors and non-survivors) on the basis of 180-day death. The gender, length of ICU stay and admission for emergence between the two groups were not different. The survivor group had a longer length of stay in the hospital. However, a higher percentage of patients exercised oliguria, shock and need for RRT in the non-survivor group. They also had a higher need for mechanical ventilation $(\mathrm{P}<0.009)$, more failed organs $(\mathrm{P}<0.000)$ and higher mean values of the APACHE II score than the survival patient group $(\mathrm{P}<0.000)$. Urinary concentration of KIM-1 and NGAL increased significantly in the non-survivor group and there was clear difference in the urinary concentrations between those in the survivor group and the non-survivor group. uNGAL and uKIM-1 concentrations increased in tandem with APACHE II score quartiles $(\mathrm{P}<0.001$; Figs. 1 and 2$)$. The overall mortality among patients studied was $39.1 \%$ (36 out of 92 patients). The primary source of sepsis was intra-abdominal (45.7\%) and pulmonary thoracic $(31.5 \%)$.

To test whether UKIM and UNGAL levels predicts 180-day mortality, we initially performed univariate Cox proportional hazards analyses incorporating multiple demographic, clinical and laboratory variables (Table III). All variables found to be statistically significant at a $5 \%$ level in the univariate analysis (APACHE II score, oliguria, failure of organs $\geq 2, \mathrm{RRT}, \mathrm{Cr}$, 
Table II. Baseline demographic characteristics of the patient population by vital status at 180 days.

\begin{tabular}{|c|c|c|c|}
\hline & Survival $(\mathrm{n}=56)$ & Non-survival $(\mathrm{n}=36)$ & P-value \\
\hline Age (years) & $55.0 \pm 7.7$ & $59.5 \pm 8.3$ & 0.090 \\
\hline Males/females & $25 / 31$ & $21 / 15$ & 0.285 \\
\hline \multicolumn{4}{|l|}{ Stage at diagnosis (RIFLE) } \\
\hline $\mathrm{R}$, risk of renal dysfunction & 16 & 3 & 0.000 \\
\hline I, Injury to the kidney & 28 & 14 & 0.000 \\
\hline $\mathrm{F}$, failure of kidney function & 8 & 23 & 0.000 \\
\hline Length of ICU stay & $9.0 \pm 7.2$ & $7.7 \pm 3.8$ & 0.323 \\
\hline Length of hospital stay & $36.3 \pm 15.6$ & $18.4 \pm 6.7$ & 0.000 \\
\hline Emergence & $18(32.1 \%)$ & $17(47.2 \%)$ & 0.188 \\
\hline Shock & $28(50.0 \%)$ & $29(80.6 \%)$ & 0.004 \\
\hline Mechanical ventilation & $18(32.1 \%)$ & $23(63.9 \%)$ & 0.005 \\
\hline APACHE II score & $18.5 \pm 4.1$ & $23.9 \pm 4.9$ & 0.000 \\
\hline Oliguria & $24(42.9 \%)$ & $23(63.9 \%)$ & 0.057 \\
\hline RRT & $2(3.6 \%)$ & $7(19.4 \%)$ & 0.026 \\
\hline Failure of organs $\geq 2$ & $22(39.3 \%)$ & $24(66.7 \%)$ & 0.018 \\
\hline Serum creatinine $(\mathrm{mmol} / \mathrm{l})$ & $218.7 \pm 66.5$ & $258.3 \pm 77.1$ & 0.010 \\
\hline $\mathrm{CrCL}$ & $30.2 \pm 10.2$ & $24.3 \pm 9.2$ & 0.007 \\
\hline KIM-1 (ng/mg) & $7.9 \pm 4.1$ & $8.9 \pm 4.9$ & 0.288 \\
\hline NGAL (ng/mg) & $308.9 \pm 155.2$ & $539.0 \pm 287.6$ & 0.000 \\
\hline
\end{tabular}

Numbers are counts and percentages $(\%)$ for categorical variables, mean and standard deviation (SD) for continuous variables. P-values for difference between groups. APACHE II, Acute Physiology and Chronic Health Evaluation II; RRT, renal replacement therapy; KIM-1, kidney injury molecule-1; NGAL, neutrophil gelatinase-associated lipocalin; CrCL, by Cockcroft-Gault formula.

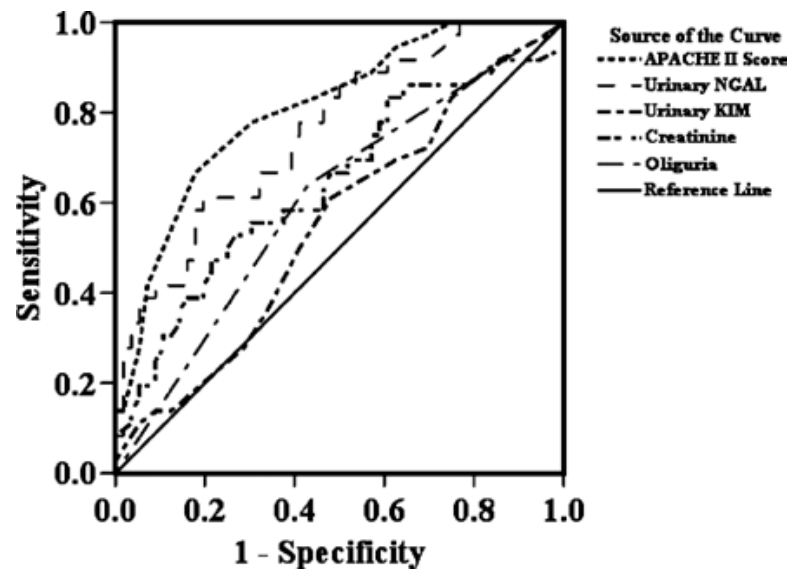

Figure 1. Receiver operating characteristic (ROC) curve.

uKIM, uNGAL, CrCL and Shock) were subjected to multivariate Cox regression analysis. As a result, the APACHE II score $(\mathrm{P}=0.014)$ and $\mathrm{uNGAL}(\mathrm{P}=0.015)$ were identified as independent predictors of 180-day mortality. When visualized by Kaplan-Meier curves, lower event-free survival was shown in patients with higher uNGAL concentrations compared to those with lower uNGAL concentrations (Fig. 3). A similar phenomenon was observed in the APACHE II score (Fig. 4). On the other hand, there was no statistical difference in eventfree survival between patients with and without higher serum creatinine, creatinine clearance and UKIM-1 (data not shown). Moreover, after combining the UNGAL and APACHE II score,
A

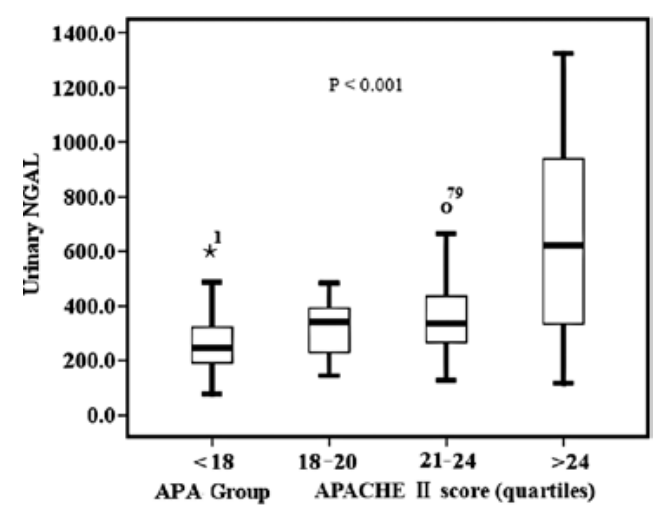

B

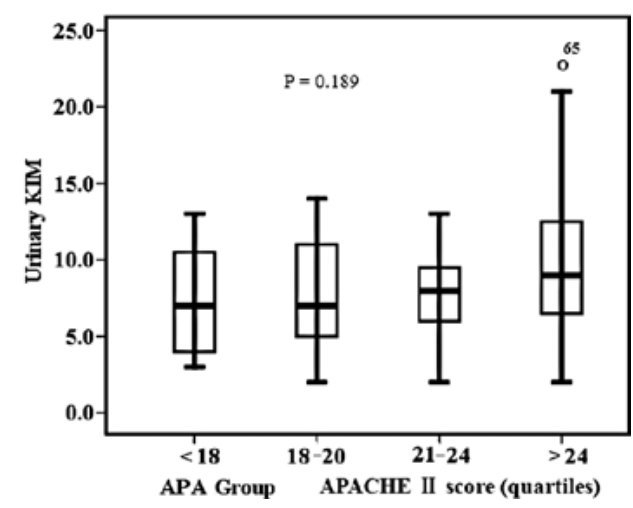

Figure 2. (A) Urinary neutrophil gelatinase-associated lipocalin (uNGAL) activity and (B) urinary kidney injury molecule-1 (uKIM-1) level stratified by the Acute Physiology and Chronic Health Evaluation (APACHE) II score quartile. $\mathrm{P}<0.001$ for NGAL activity and $\mathrm{P}=0.189$ for KIM-1 level, by the Kruskal-Wallis test. The box and whisker plots show the 10th, 25th, 50th (median), 75th and 90th percentile values. 
Table III. Univariate and multivariable Cox regression analysis for the 180-day mortality rate.

\begin{tabular}{lccc}
\hline \multirow{2}{*}{ Variables } & \multicolumn{1}{c}{ Univariate analysis } & & Multivariate analysis \\
\cline { 2 - 3 } & \multicolumn{1}{c}{ HR $(95 \% \mathrm{CI})$} & P-value & HR (95\% CI) \\
\hline APACHE II & $1.181(1.112-1.254)$ & 0.000 & 0.005 \\
Age (group) & $1.755(1.185-2.600)$ & 0.183 & \\
Gender & $1.570(0.809-3.047)$ & 0.042 & \\
Oliguria & $2.030(1.027-4.011)$ & 0.018 & \\
Failure of organs $\geq 2$ & $2.308(1.154-4.618)$ & 0.169 & \\
Emergence & $1.584(0.823-3.048)$ & 0.013 & \\
RRT & $2.886(1.253-6.644)$ & 0.017 & \\
Cr & $1.005(1.001-1.010)$ & 0.300 & \\
KIM-1 & $1.039(0.967-1.116)$ & 0.000 & \\
NGAL & $1.003(1.002-1.004)$ & 0.011 & \\
CrCL & $0.955(0.922-0.989)$ & 0.007 & 0.006 \\
Shock & $3.112(1.362-7.113)$ & 0.007 & \\
MV & $2.573(1.302-5.084)$ & $.004)$
\end{tabular}

APACHE II, Acute Physiology and Chronic Health Evaluation II; RRT, renal replacement therapy; KIM-1, kidney injury molecule-1; NGAL, neutrophil gelatinase-associated lipocalin; CrCL, by Cockcroft-Gault formula.

A

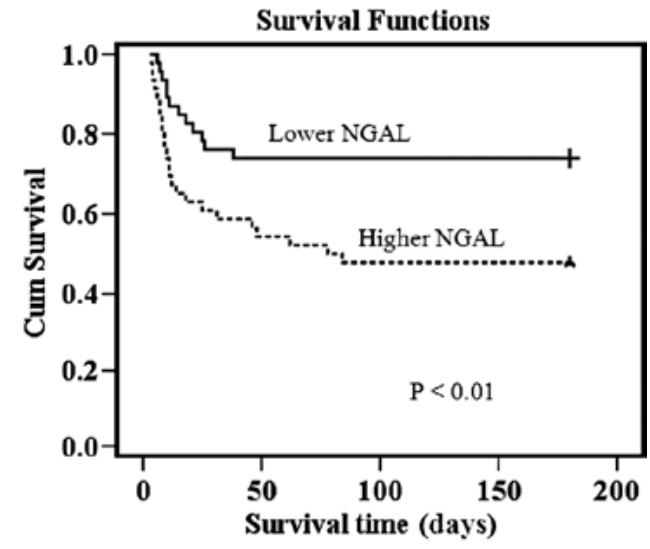

B

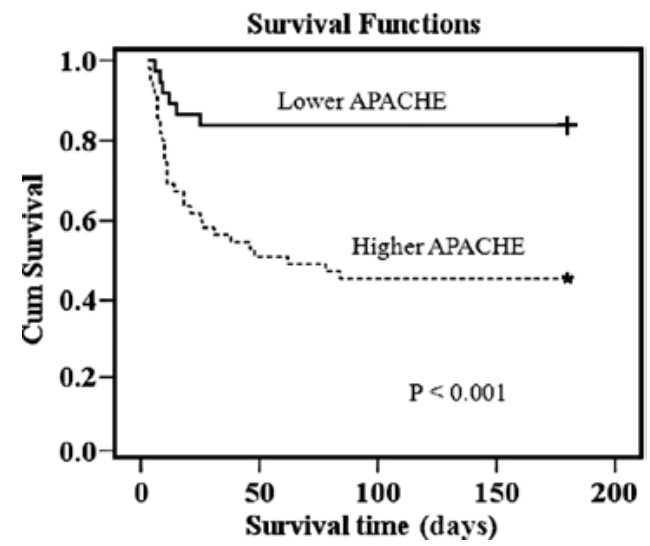

Figure 3. Probability of survival of all septic-AKI patients according to (A) NGAL and (B) APACHE levels (higher vs. lower values). For NGAL, mortality at 180 days 26.1 vs. $52.2 \%$; log rank test, $\mathrm{P}<0.01$; for APACHE, mortality at 180 days 16.2 vs. $54.4 \%$; log rank test, $\mathrm{P}<0.001$.

three separate risk groups yielded low, medium and high 180-day mortality (Fig. 4). Compared to the first group, the risk of death increased significantly with each group.

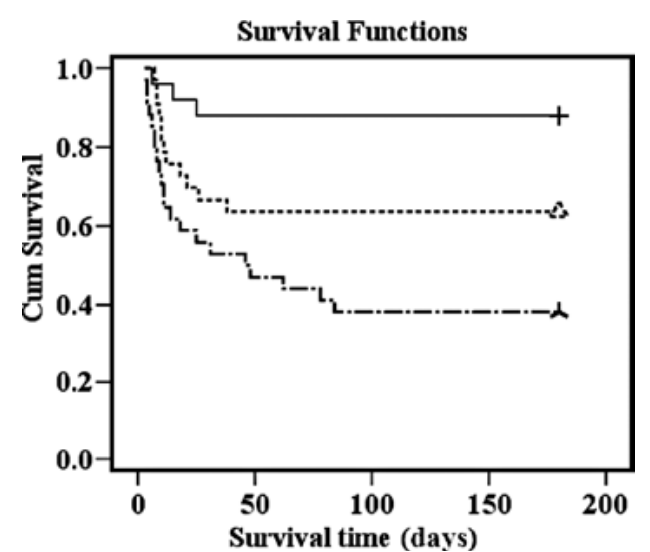

Figure 4. One hundred and eighty-day survival by combining NGAL and APACHE II score. All-cause mortality at 180 days: $12 \%$ for first tertile (upper line), $36.4 \%$ for second tertile (middle line) and $61.8 \%$ for third tertile (lower line). P-values (log-rank test) for difference between tertiles: first vs. second, $\mathrm{P}=0.044$; second vs. third, $\mathrm{P}=0.046$; first vs. third, $\mathrm{P}<0.001$.

Table IV displays the area under the ROC curve (AUC) of selected clinical and urinary predictor variables for the 180-day death. The APACHE II score, a composite score of 15 clinical variables, provided the best discrimination compared to UNGAL for the 180-day death $(0.785,0.694-0.876)$. The uNGAL level performed better than serum creatinine or oliguria, two traditional clinical parameters. However, uKIM-1 showed slightly better discrimination compared to serum creatinine.

\section{Discussion}

Sepsis is an serious problem in ICU patients, not only because of its high incidence, but also because of the high mortality associated with this condition. It is a well-known fact that sepsis is an important risk factor for the development of AKI, and mortality in this specific subgroup of AKI patients is consider- 
Table IV. AUC of several variables for the prediction of the outcome of 180-day mortality.

\begin{tabular}{lcccc}
\hline & & & \multicolumn{2}{c}{$\operatorname{Exp(B)} 95 \%$ CI } \\
\cline { 4 - 5 } Parameter & AUC & P-value & $\begin{array}{l}\text { Lower } \\
\text { bound }\end{array}$ & $\begin{array}{c}\text { Upper } \\
\text { bound }\end{array}$ \\
\hline APACHE II score & 0.808 & 0.000 & 0.718 & 0.897 \\
NGAL & 0.759 & 0.000 & 0.660 & 0.858 \\
KIM-1 & 0.550 & 0.421 & 0.430 & 0.670 \\
Cr & 0.642 & 0.022 & 0.522 & 0.761 \\
Oliguria & 0.605 & 0.090 & 0.487 & 0.724 \\
\hline
\end{tabular}

AUC, area under the receiver operating characteristic curve (ROC).

ably higher than in others (15-18). Such a high incidence and marked disparity in outcome implies a need for increasing our understanding of the pathophysiology, diagnosis and treatment options for septic AKI (19). Animal data indicate that septic AKI may have distinct pathophysiologic features that differ from ischemia/toxin-induced kidney injury (20). For example, in a mammalian model of $E$. coli-induced hyperdynamic sepsis, renal blood flow was increased nearly $300 \%$ from baseline at $48 \mathrm{~h}(21)$. This renal hyperemia was evident despite severe reductions in kidney function. This phenomenon has been similarly described in septic humans (22-24).

These observations may have implications for the critically ill septic patient. Firstly, unique patterns of urine biochemistry and/or biomarkers may exist in septic AKI that can be detected early, guide therapy, aid in clinical decision-making (early renal support) and/or provide prognostic information (renal recovery) (25). Secondly, emerging literature has challenged the conventional paradigm concerning the value of urine biochemistry and derived indices in septic AKI (26-28). This widely accepted paradigm for classifying AKI (i.e., pre-renal azotemia or acute tubular necrosis) may be flawed in septic AKI, and novel methods for evaluation are required $(25,28)$.

To the best of our knowledge, this is the first study to investigate prognostic factors among patients with septic AKI in China, especially using kidney injury makers, such as uNGAL and uKIM-1. We found that the survival group patients with septic AKI, when compared to the non-survival septic AKI group, had considerable differences across numerous clinical, physiologic and laboratory parameters. Non-survival patients were generally older, had a shorter hospital stay, higher illness severity and organ dysfunction, and were more likely to have undergone mechnical ventilation therapy. These differences have likewise been described in prior studies (29-31). We also found that the uNGAL level increased in tandem with APACHE II score quartiles and was a strong predictor of mortality compared to creatinine or uKIM-1. Mortality increased significantly with every tertile of the compilation of UNGAL and APACHE II score. Consequently, uNGAL enables stratification of patients with septic AKI according to risk of death, and may help in directing intensified treatment efforts to high-risk groups.

NGAL is a $25-\mathrm{kDa}$ protein that is expressed at very low concentrations in several human tissues, including kidney, lungs and gastrointestinal tract $(6,32)$. It is highly up-regulated in injured epithelial cells. The detection of NGAL has been described in several studies as an early sensitive biomarker of AKI that precedes the lag-time effect needed to detect abnormalities in traditional measures of kidney function $(9,14,33-39)$. Recent studies have demonstrated the utility of early NGAL measurements for predicting clinical outcomes of AKI. In cardiac surgery, the 2-h uNGAL levels measured by ARCHITECT $^{\circledR}$ analyzer highly correlated with duration and severity of AKI, length of hospital stay, dialysis requirement and death (40). In a multicenter study of children with diarrheaassociated hemolytic uremic syndrome, uNGAL obtained early during the hospitalization predicted the severity of AKI and dialysis requirement with high sensitivity (34). Early uNGAL levels were also predictive of duration of AKI (AUC 0.79) in a heterogeneous cohort of critically ill subjects (41). Emerging literature suggests that uNGAL is also a marker of chronic kidney disease (CKD) and its severity (42). In subjects with $\mathrm{CKD}$, due to glomerulonephritis, uNGAL levels were found to be elevated and significantly correlated with serum creatinine, GFR and proteinuria (43). In patients with autosomal dominant polycystic kidney disease, uNGAL measurements correlated with residual GFR and severity of cystic disease $(44,45)$, raising the possibility of NGAL representing a non-invasive screening tool for the detection of tubulo-interstitial disease.

Reliable identification of renal dysfunction is a key issue in identifying patients at risk of death. In clinical practice, however, assessment of renal function is dependent on readily available methods. The relationship between creatinine and GFR is non-linear and creatinine is an insensitive measure for detecting small decreases in GFR (46). Creatinine concentrations are also affected by factors, such as age, gender, muscle mass and diet. As a sensitive marker of renal function, uNGAL probably overcomes many of the shortcomings of the more commonly used measures of renal function in the setting of AKI. Consequently, we suggest that the preferred marker of renal function with the strongest prognostic value for patients with AKI is uNGAL.

We acknowledge that increased mortality with higher levels of NGAL largely reflects the association with renal dysfunction. Whether the multivariable prognostic value of NGAL is solely due to its superior ability to estimate the true renal function and detect real changes in GFR is not clear. We consider NGAL a possible marker of impaired renal perfusion that reflects early end organ failure in patients with AKI. However, we cannot exclude that NGAL affects prognosis by mechanisms unrelated to renal function. Further studies are required to increase our knowledge about the multivariable effect on mortality in septic AKI and possible underlying mechanisms.

We demonstrated the ability of NGAL to distinguish patients with septic AKI having low, median and high risk of death in combination with the APACHE II score. The difference in mortality between each group is marked and statistically highly significant. This finding provides clinicians with a simple tool in risk stratification, which is essential in the management of septic AKI.

There are limitations to our study. First, this was a prospective study with a small number of patients and relatively short follow-up, which may be associated with the observed lack of 
a significant difference between groups. Further studies with a larger number of patients and longer follow-up are required to evaluate the usefulness of the UNGAL level for predicting death during follow-up after AKI. Second, we did not include a septic non-AKI control group for comparison. We also did not study the cause of death in our patients and therefore did not obtain data on the mechanisms linking renal function impairment and outcomes.

In conclusion, our study provides evidence that UNGAL is a sensitive marker of renal insufficiency in septic AKI patients, correlates with APACHE II score and has an independent prognostic significance for survival. Notably, its combination with APACHE II score produces a prognostic model which separates newly-diagnosed septic AKI patients into three risk groups: low-, median- and high-risk groups. Therefore, its measurement provides important information for septic AKI patients. According to our data, routine assessment of uNGAL in septic AKI should be recommended, while its combination with APACHE II score will be a useful tool for the prognosis of septic AKI patients.

\section{Acknowledgements}

The authors gratefully acknowledge the assistance of the medical and nursing staff of the ICU and of the Nephrology Section of the Xiangya Hospital.

\section{References}

1. Riedemann NC, Guo RF and Ward PA: The enigma of sepsis. J Clin Invest 112: 460-467, 2003.

2. Rangel-Frausto MS, Pittet D, Costigan M, et al: The natural history of the systemic inflammatory response syndrome (SIRS): a prospective study. JAMA 273: 117-123, 1995.

3. Uchino S, Kellum JA, Bellomo R, et al: Acute renal failure in critically ill patients: a multinational, multicenter study. JAMA 294: 813-818, 2005.

4. Angus DC, Linde-Zwirble WT, Lidicker J, et al: Epidemiology of severe sepsis in the United States: analysis of incidence, outcome and associated costs of care. Crit Care Med 29: 1303-1310, 2001.

5. Edelstein CL and Schrier RW: Pathophysiology of ischemic acute renal failure. In: Schrier RW (ed): Diseases of the Kidney and Urinary Tract. 7th edition. Vol. 2. Lippincott Williams \& Wilkins, Philadelphia, pp1041-1069, 2001.

6. Cowland JB and Borregaard N: Molecular characterization and pattern of tissue expression of the gene for neutrophil gelatinaseassociated lipocalin from humans. Genomics 45: 17-23, 1997.

7. Cowland JB, Sorensen OE, Schested M, et al: Neutrophil gelatinase-associated lipocalin is up-regulated in human epithelial cells by IL-1 $\beta$ but not by TNF- $\alpha$. J Immunol 171: 6630-6639, 2003

8. Mishra J, Mori K, Ma Q, et al: Amelioration of ischemic acute renal injury by neutrophil gelatinase-associated lipocalin. J Am Soc Nephrol 15: 3073-3082, 2004

9. Mishra J, Dent C, Tarabishi R, et al: Neutrophil gelatinase-associated lipocalin (NGAL) as a biomarker for acute renal injury after cardiac surgery. Lancet 365: 1231-1238, 2005.

10. Boldt J, Brenner T, Lehmann A, et al: Is kidney function altered by the duration of cardiopulmonary bypass? Ann Thorac Surg 75: 906-912, 2003

11. Bellomo R and Ronco C: Acute renal failure - definition, outcome measures, animal models, fluid therapy and information technology needs: the Second International Consensus Conference of the Acute Dialysis Quality Initiative (ADQI) Group. Crit Care 8: R204-R212, 2004

12. Levy MM, Fink MP, Marshall JC, et al: 2001 SCCM/ESICM/ ACCP/ATS/SIS international sepsis definitions conference. Crit Care Med 31: 1250-1256, 2003.

13. Charlson ME, Pompei P, Ales KL, et al: A new method of classifying prognostic comorbidity in longitudinal studies: development and validation. J Chronic Dis 40: 373-383, 1987.
14. Nickolas TL, O'Rourke MJ, Yang J, et al: Sensitivity and specificity of a single emergency department measurement of urinary neutrophil gelatinase-associated lipocalin for diagnosing acute kidney injury. Ann Intern Med 148: 810-819, 2008.

15. Slot C: Plasma creatinine determination: a new and specific Jaffe reaction method. Scand J Clin Lab Invest 17: 381-387, 1965.

16. Brivet F, Kleinknecht D, Loirat P, et al: The French study group on acute renal failure: acute renal failure in intensive care units causes, outcome, and prognostic factors on hospital mortality: a prospective, multicenter study. Crit Care Med 24: 192-198, 1996.

17. Liano F, Junco E, Pascual J, et al: The spectrum of acute renal failure in the intensive care unit compared to that seen in other settings. Kidney Int 53 (Suppl 66): 16-24, 1998.

18. Neveu H, Kleinknecht $\mathrm{D}$, Brivet F, et al: Prognostic factors in acute renal failure due to sepsis. Results of a prospective multicentre study. Nephrol Dial Transplant 11: 293-299, 1996.

19. Cole L, Bellomo R, Silvester W, et al: A prospective, multi-center study of the epidemiology, management, and outcome of severe acute renal failure in a 'closed' ICU system. Am J Respir Crit Care Med 162: 191-196, 2000.

20. Wan L, Bagshaw SM, Langenberg C, et al: Pathophysiology of septic acute kidney injury: what do we really know? Crit Care Med 36: S198-S203, 2008.

21. Wan L, Bellomo R, di Giantomasso D, et al: The pathogenesis of septic acute renal failure. Curr Opin Crit Care 9: 496-502, 2003.

22. Langenberg C, Wan L, Egi M, et al: Renal blood flow in experimental septic acute renal failure. Kidney Int 69: 1996-2002, 2006.

23. Brenner M, Schaer GL, Mallory DL, et al: Detection of renal blood flow abnormalities in septic and critically ill patients using a newly designed indwelling thermodilution renal vein catheter. Chest 98: 170-179, 1990.

24. Lucas CE, Rector FE, Werner M, et al: Altered renal homeostasis with acute sepsis. Clinical significance. Arch Surg 106: 444-449, 1973.

25. Rector F, Goyal S, Rosenberg IK, et al: Renal hyperemia is associated with clinical sepsis. Surg Forum 23: 51-53, 1972.

26. Bellomo R, Bagshaw S, Langenberg C, et al: A flawed paradigm in critically ill septic patients? Contrib Nephrol 156: 1-9, 2007.

27. Bagshaw SM, Langenberg C, Haase M, et al: Urinary biomarkers in septic acute kidney injury. Intensive Care Med 33: 1285-1296, 2007.

28. Bagshaw SM, Langenberg C, Wan L, et al: A systematic review of urinary findings in experimental septic acute renal failure. Crit Care Med 35: 1592-1598, 2007.

29. Schrier RW: Urinary indices and microscopy in sepsis-related acute renal failure. Am J Kidney Dis 48: 838-841, 2006.

30. Bagshaw SM, George C and Bellomo R: Early acute kidney injury and sepsis: a multicentre evaluation. Crit Care 12: R47, 2008.

31. Bagshaw SM, Uchino S, Bellomo R, et al: Septic acute kidney injury in critically ill patients: clinical characteristics and outcomes. Clin J Am Soc Nephrol 2: 431-439, 2007.

32. Bagshaw SM, Lapinsky S, Dial S, et al: Acute kidney injury in septic shock: clinical outcomes and impact of duration of hypotension prior to initiation of antimicrobial therapy. Intensive Care Med 35: 871-881, 2009.

33. Kjeldsen L, Cowland JB and Borregaard N: Human neutrophil gelatinase-associated lipocalin and homologous proteins in rat and mouse. Biochim Biophys Acta 1482: 272-283, 2000.

34. Bennett M, Dent CL, Ma Q, et al: Urine NGAL predicts severity of acute kidney injury after cardiac surgery: a prospective study. Clin J Am Soc Nephrol 3: 665-673, 2008.

35. Dent CL, Ma Q, Dastrala S, et al: Plasma neutrophil gelatinaseassociated lipocalin predicts acute kidney injury, morbidity and mortality after pediatric cardiac surgery: a prospective uncontrolled cohort study. Crit Care 11: R127, 2007.

36. Haase-Fielitz A, Bellomo R, Devarajan P, et al: Novel and conventional serum biomarkers predicting acute kidney injury in adult cardiac surgery: a prospective cohort study. Crit Care Med 37: 553-560, 2009.

37. Mishra J, Mori K, Ma Q, et al: Neutrophil gelatinase-associated lipocalin: a novel early urinary biomarker for cisplatin nephrotoxicity. Am J Nephrol 24: 307-315, 2004.

38. Wagener G, Jan M, Kim M, et al: Association between increases in urinary neutrophil gelatinase-associated lipocalin and acute renal dysfunction after adult cardiac surgery. Anesthesiology 105: 485-491, 2006.

39. Bachorzewska-Gajewska H, Malyszko J, Sitniewska E, et al: Neutrophil-gelatinase-associated lipocalin and renal function after percutaneous coronary interventions. Am J Nephrol 26: 287-292, 2006 
40. Haase M, Haase-Fielitz A, Bellomo R, et al: Sodium bicarbonate to prevent increases in serum creatinine after cardiac surgery: a pilot double-blind, randomized controlled trial. Crit Care Med 37: 39-47, 2009.

41. Trachtman H, Christen E, Cnaan A, et al: Urinary neutrophil gelatinase-associated lipocalcin in D+HUS: a novel marker of renal injury. Pediatr Nephrol 21: 989-994, 2006.

42. Zappitelli M, Washburn KM, Arikan AA, et al: Urine NGAL is an early marker of acute kidney injury in critically ill children. Crit Care 11: R84, 2007.

43. Nickolas TL, Barasch J and Devarajan P: Biomarkers in acute and chronic kidney disease. Curr Opin Nephrol Hypertens 17: 127-132, 2008.
44. Bolignano D, Coppolino G, Campo S, et al: Urinary neutrophil gelatinase-associated lipocalin (NGAL) is associated with severity of renal disease in proteinuric patients. Nephrol Dial Transplant 23: 414-416, 2008.

45. Bolignano D, Coppolino G, Campo S, et al: Neutrophil gelatinase-associated lipocalin in patients with autosomal-dominant polycystic kidney disease. Am J Nephrol 27: 373-378, 2007.

46. Stevens LA, Coresh J, Greene T, et al: Assessing kidney function measured and estimated glomerular filtrationrate. N Engl J Med 354: 2473-2483, 2006. 